\title{
Near-Capacity Turbo Coded Soft-decision Aided DAPSK/Star-QAM for Amplify-and-Forward based Cooperative Communications
}

\author{
Dandan Liang, Student Member, IEEE, Soon Xin Ng, Senior Member, IEEE, and Lajos Hanzo, Fellow, IEEE
}

\begin{abstract}
Multilevel Differential Amplitude and Phase-Shift Keying (DAPSK) schemes do not require any channel estimation, which results in low complexity. In this treatise we derive the soft-output probability formulas required for a soft-decision based demodulation of high-order DAPSK, in order to facilitate iterative detection by exchanging extrinsic information with an outer Turbo Code (TC). Furthermore, when the TC block size is increased, the system operates closer to the channel capacity. Compared to the identical-throughput TC assisted 64ary Differential Phase-Shift Keying (64-DPSK) scheme, the 4-ring based TC assisted 64-ary DAPSK arrangement has a powerefficiency improvement of $2.3 \mathrm{~dB}$ at a bit error rate (BER) of $10^{-5}$. Furthermore, when the TC block size is increased, the system operates closer to the channel capacity. More specifically, when using a TC block length of 400 modulated symbols, the 64 DAPSK $(4,16)$ scheme is $7.56 \mathrm{~dB}$ away from its capacity curve, while it had a reduced gap as low as $2.25 \mathrm{~dB}$, when using a longer TC block length of 40000 modulated symbols. Finally, as a novel application example, the soft-decision M-DAPSK scheme was incorporated into an Amplify-and-Forward (AF) based cooperative communication system, which attains another 4.5 dB SNR improvement for a TC block length of 40000 modulated symbols.
\end{abstract}

Index Terms-Soft-decision, iterative detection, DAPSK, turbo coding, amplify-and-forward (AF) relaying protocol, correlated Rayleigh fading channel, near-capacity transceivers.

\section{INTRODUCTION}

D IFFERENTIALLY encoded non-coherently detected modulation techniques have a low complexity, since they do not require any Channel State Information (CSI). Differential Phase Shift Keying (DPSK) is a classic differentially encoded modulation scheme, which only takes the phase information into account. Upon increasing the number of amplitude-rings, the concept of Differential Amplitude and Phase-Shift Keying (DAPSK) was conceived, which can also be interpreted as an extension of the DPSK scheme [2][5]. More specifically, both the amplitude and the phase information are differentially encoded. Owing to its robustness

Paper approved by Q. S. T. Quek, the Editor for Heterogeneous Networks and Green Communications of the IEEE Communications Society. Manuscript received November 20, 2011; revised February 14, 2012 and July 2, 2012.

The authors are with the Communications, Signal Processing and Control Research Group, University of Southampton, SO17 1BJ, U.K. (e-mail: \{dl4e08, sxn, lh\}@ecs.soton.ac.uk).

The financial support of the EPSRC UK under the auspices of the ChinaUK Science Bridge, the India-UK Advanced Technology Centre and the EUs 7th Framework Programme (FP7/2007-2013) under the auspices of the CONCERTO project (288502) is gratefully acknowledged.

Some of the earlier results have been published in [1].

Digital Object Identifier 10.1109/TCOMM.2012.09.110786 to false phase-locking of the carrier-recovery and due to its better performance than that of the identical-throughput DPSK scheme, there has been growing interest in differentially encoded multilevel modulation schemes conceived for achieving a high data-rate [2]-[5]. In this paper, the notation M-DAPSK $\left(M_{a}, M_{p}\right)$ associated with $M_{a}$ amplitudes and $M_{p}$ different phases is used, which may also be written as $M_{a^{-}}$ DASK $/ M_{p}$-DPSK. The twin-ring based DAPSK scheme is also often referred to as Star-QAM [6].

When channel coding is incorporated into $\mathrm{M}$ DAPSK $\left(M_{a}, M_{p}\right)$ as in [6], its performance remains far from the corresponding detection-dependent Discreteinput Continuous-output Memoryless Channel's (DCMC) capacity owing to the employment of hard-decision based demodulation. Hence, a variety of techniques have been proposed in the literature [7]-[10] for overcoming the performance loss imposed by employing hard-decision M-DAPSK $\left(M_{a}, M_{p}\right)$. More specifically, a sub-optimal yet high-complexity soft Viterbi decoding metric was proposed in [7], [8], [10], which requires a high SNR and a slowly fading channel. In order to reduce the high computational complexity of the receiver, the authors of [9] quantized the received signals as part of the demodulation process based on the maximum likelihood sequence estimator derived in [8]. As another approach of reducing the complexity, the idea of decoupling the joint amplitude and phase detection, the Bit Metric of Iterative a posteriori probability (APP) Decoders (BMIAD), was proposed as an improvement by [10]. More specifically, the BMIAD scheme assumes that the channel SNR is high enough to ensure that the channel noise can be ignored [10, Eq. (37)]. However, we are interested in achieving a high integrity at low SNRs, when designing near-capacity coding schemes. An attractive soft-decision aided demodulator capable of reliably operating right across the entire SNR region was proposed for a two-ring based DAPSK scheme in [11].

In this contribution, we extend the soft-decision demodulation algorithm derived for the twin-ring Star-QAM [11] to multiple-ring based DAPSK schemes. Turbo Coding (TC) [12] is employed in our DAPSK scheme in order to achieve further improvements. The important technical breakthrough of TC was proposed in [12], [13], where exchanging extrinsic information between two Recursive Systematic Convolutional (RSC) decoders was shown to achieve a substantial performance improvement. The appealing iterative decoding of 
concatenated codes has inspired numerous researchers to aim for achieving a near-capacity performance in diverse system contexts [14]. Moreover, the concept of EXtrinsic Information Transfer (EXIT) charts was proposed in [15] for analysing the convergence behaviour of turbo detection. The EXIT curve [15] of a TC coder was found to have a better match with that of the M-DAPSK $\left(M_{a}, M_{p}\right)$ demapper, than the match between the BICM-ID and M-DAPSK $\left(M_{a}, M_{p}\right)$ demapper EXIT-curves. Since having a smaller area between these two EXIT curves indicates that the system operates closer to the achievable capacity [14], the TC aided M-DAPSK $\left(M_{a}, M_{p}\right)$ arrangement approaches the channel capacity more closely than the BICM-ID aided M-DAPSK scheme.

Furthermore, cooperative communications [16], [17] is capable of supporting the users either at an improved integrity or throughput in wireless networks. In contrast to the decodeand-forward (DF) protocol, neither demodulation nor decoding is required at the relay by the non-regenerative amplify-andforward (AF) protocol. Hence, AF relaying is considered as a beneficial cooperative techniques in low-complexity scenarios. Most of the previous contributions on relay aided systems assume that the destination is capable of acquiring perfect CSI for all transmission links in order to carry out coherent detection [17]. However, in rapidly fading environments, the CSI cannot be accurately estimated at the destination. DAPSK constitutes an ideal candidate for mobile relaying aided wireless communications, since it is unrealistic to expect that the relay altruistically estimates the source-relay channel for both complexity and information security reasons [14]. Despite a wealth of past studies on the employment of DPSK in AF based relaying schemes [18], [19], the single-ring based DPSK scheme yields poorer performance than DAPSK, when the modulation alphabet size is large. As an improvement, the DAPSK [20] was proposed for an AF relaying system communicating over independent Rician fading channels. As a novel application example, in this contribution, we have amalgameted the soft-decision DAPSK aided channel coding scheme with an AF based relaying system transmitting over correlated Rayleigh fading channels.

Our novel contributions are as follows:

1) We uesed the existing BMIAD 16-DAPSK $(2,8)$ scheme of [10] as a benchmark for our proposed 16DAPSK $(2,8)$ schemes.

2) We derived the soft-decision demodulation probability formulas for M-DAPSK $\left(M_{a}, M_{p}\right)$ schemes, which have more than two concentric PSK rings.

3) We quantified the EXIT-chart-based throughput of $M$ DAPSK $\left(M_{a}, M_{p}\right)$ systems and showed that our TCaided M-DAPSK $\left(M_{a}, M_{p}\right)$ schemes are capable of approaching the achievable channel capacity.

4) We then conceived a novel soft-decision DAPSK aided AF based cooperative systems, which completely dispenses with channel estimation.

The structure of this paper is as follows. In Section II both the M-DAPSK concepts will be highlighted. The corresponding simulation results, EXIT chart analysis and achievable capacity will be discussed in Section III. Finally, our conclusions will be presented in Section IV.

\section{System Model And AnAlysis}

Fig. 1 presents the simplified schematic of our near-capacity TC aided M-DAPSK $\left(M_{a}, M_{p}\right)$ scheme, where the number of constellation points is $\mathrm{M}=M_{a} \cdot M_{p}=2^{\mathrm{m}}$, while the number of amplitudes is $M_{a}=2^{m_{a}}$ and the number of phases per amplitude-circle is $M_{p}=2^{m_{p}}$. A sequence of coded symbols is generated by a sequence of a rate-1/2 TC encoded information symbols. Out of the total number of modulated bits per symbol, which is $\mathrm{m}, m_{a}$ bits will be assigned for the selection of the Phase-Shift-Keying (PSK) amplitude ring, while the remaining $\left(m_{p}=\mathrm{m}-m_{a}\right)$ bits will be used for selecting the phase of the complex-valued M-DAPSK $\left(M_{a}, M_{p}\right)$ symbol $x_{k}$, where the subscript $k$ denotes the symbol index. The near-capacity TC aided MDAPSK $\left(M_{a}, M_{p}\right)$ system will be illustrated in Section II-A and Section II-B. Furthermore, our soft-decision M-DAPSK aided AF relaying protocol dispensing with channel estimation will be detailed in Section II-C.

As shown in Fig. 1, the TC-encoded M-DAPSK $\left(M_{a}, M_{p}\right)$ symbol is corrupted by both the Rayleigh fading channel and the Additive White Gaussian Noise (AWGN), when it is transmitted to the receiver. Then, based on the received sequence $\left\{y_{k}\right\}$ but without exploiting any CSI, we exchange extrinsic information between the M-DAPSK soft demapper and the TC decoder to accomplish iterative detection.

\section{A. M-DAPSK Mapper}

The M-DAPSK $\left(M_{a}, M_{p}\right)$ mapper shown in Fig. 1 consists of two components, namely the amplitude selector and a conventional $M_{p}$-level DPSK $\left(M_{p}\right.$-DPSK) mapper. Note that $M_{p}$-DPSK is formed by the $M_{p}$-PSK mapper and the differential encoder. It is worth noting that similar to any classic DPSK scheme, we insert a reference symbol at the beginning of each differentially encoded transmission frame before the M-DAPSK $\left(M_{a}, M_{p}\right)$ mapper. Additionally, the 16-DAPSK $(2,8)$ scheme and the 64-DAPSK $(4,16)$ scheme were used as examples for illustrating the philosophy of our proposed soft-decision based demapper.

1) Amplitude Selection: $m_{a}$ bits are used for selecting the amplitude of the PSK ring, $a_{k}=\varrho\left(b_{k}\right)$, where $\varrho\left(b_{k}\right)$ represents the function mapping the $m_{a}$-bit symbol $b_{k}$ to the amplitude $a_{k}$ under the combined constraint of $0 \leq b_{k} \leq$ $\left(M_{a}-1\right)$ and $b_{k}=\left(b_{k-1}+f_{k}\right) \bmod M_{a}$, with $f_{k}=$ $\sum_{i=m_{p}}^{\mathrm{m}-1} 2^{\left(i-m_{p}\right)} c_{\mathrm{m} k+i}$, where $c_{\mathrm{m} k+i}, 0 \leq \mathrm{m} k+i \leq\left(N_{c}-1\right)$, is the binary coded sequence of length $N_{c}$.

For the 16-DAPSK $(2,8)$ scheme, we have $b_{k}=\left(b_{k-1}+\right.$ $\left.c_{4 k+3}\right) \bmod 2$ for 16-DAPSK $(2,8)$, while for the 64DAPSK $(4,16)$ scheme, we have $b_{k}=\left(b_{k-1}+2 c_{6 k+5}+c_{6 k+4}\right)$ $\bmod 4$.

Note that the classic Gray Mapping [14] method is employed by the bit-to-symbol mapper.

This amplitude selection mechanism may be referred to as $M_{a}$-level Differential Amplitude Shift Keying ( $M_{a}$-DASK). After normalisation to a symbol energy of unity, we have $\varrho\left(b_{k}\right)=\alpha^{b_{k}} / \sqrt{\beta}$, here $\sum_{i=1-m_{a}}^{m_{a}-1}\left|\alpha^{b_{k}}\right|^{2}=\beta$. We use the optimum amplitude ratio of $\alpha=1.4, \beta=3.58$ for $\mathrm{M}$ $\operatorname{DAPSK}\left(4, M_{p}\right)$ and $\alpha=2.0, \beta=2.5$ for M-DAPSK (2, $M_{p}$ ) [21]. The amplitude of the reference symbol is given by $a_{-1}=\varrho(0)$. 


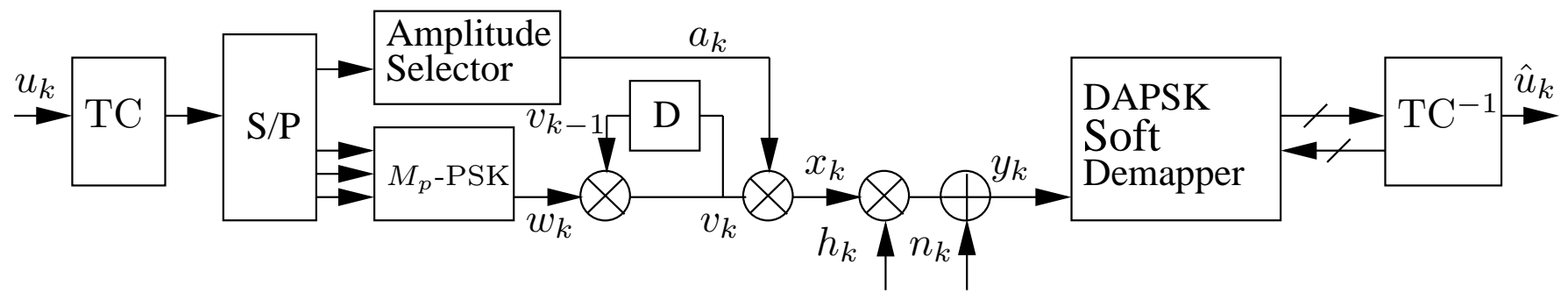

Fig. 1. The schematic of the M-DAPSK $\left(M_{a}, M_{p}\right)$ aided TC scheme, where the interleavers and de-interleavers between the encoder/decoder and mapper/demapper are not shown for simplicity.

2) Phase Selection: When we consider $m_{p}$, the $k$ th differentially encoded symbol $v_{k}$ can be expressed as:

$$
v_{k}=v_{k-1} w_{k},
$$

where $w_{k}=\mu\left(d_{k}\right)=\exp \left(j \pi d_{k} / M_{p}\right)$ is the $M_{p}$-PSK symbol obeying $d_{k}=\sum_{i=0}^{m_{p}-1} 2^{i} c_{\mathrm{m} k+i}$. More specifically, $d_{k}=4 c_{4 k+2}+2 c_{4 k+1}+c_{4 k}$ for the 16-DAPSK $(2,8)$ scheme, while $d_{k}=8 c_{6 k+3}+4 c_{6 k+2}+2 c_{6 k+1}+c_{6 k}$ for the 64DAPSK $(4,16)$ scheme and $\mu($.$) is the M_{p}$-PSK mapping function. Furthermore, $v_{k-1}$ is the $(k-1)$ st $M_{p}$-DPSK symbol and $\left|v_{k}\right|^{2}=1$. The reference symbol of the $M_{p}$-DPSK part of the constellation is given by $v_{-1}=\mu(0)=1$.

When relying on the above-mentioned amplitude and phase selection methods, the $k$ th M-DAPSK $\left(M_{a}, M_{p}\right)$ symbol may be written as:

$$
x_{k}=a_{k} v_{k}
$$

\section{B. Differential Detection}

In this sub-section, we firstly study the BMIAD, which is as our benchmark scheme. Then detail the proposed MDAPSK $\left(M_{a}, M_{p}\right)$ Soft Demapper.

1) BMIAD: The BMIAD is based on the 16-DAPSK $(2,8)$ scheme. The $k$ th received symbol may be formulated as:

$$
y_{k}=h_{k} x_{k}+n_{k}=\rho_{k} e^{j \phi_{k}} x_{k}+n_{k},
$$

where $h_{k}=\rho_{k} e^{j \phi_{k}}$ represents the non-dispersive Rayleigh fading coefficients, while $n_{k}$ represents the AWGN having a variance of $N_{0} / 2$ per dimension. Furthermore, $\rho_{k}$ and $\phi_{k}$ represent the amplitude and the phase of the fading channel, respectively.

For the APP decoder, the amplitude bit metric can be obtained from the exact a posteriori probability of $\Upsilon_{y, k}, \Delta \theta_{k}$, given $a_{k}, a_{k-1}, \psi_{k}$ and $\rho_{k}$ as [10]:

$$
\begin{array}{r}
P_{A}\left(\Upsilon_{y, k}, \Delta \theta_{k} \mid a_{k}, a_{k-1}, \psi_{k}, \rho_{k}\right) \\
\approx e^{-\frac{\left[| y _ { k - 1 } | ^ { 2 } \left(\Upsilon_{y, k}-\alpha^{\left.\left.q_{k}\right)^{2}\right]}\right.\right.}{N_{0}\left(1+\Upsilon_{y, k}^{2}\right)}} .
\end{array}
$$

where $a_{k}$ and $\psi_{k}$ denote the amplitude and phase of $x_{k}$. Moreover, $\Upsilon_{y, k}=\frac{\left|y_{k}\right|}{\left|y_{k-1}\right|}$ and $\Delta \theta_{k}=\theta_{k}-\theta_{k-1}$ represent the envelope and the phase difference, where $\theta_{k}=\angle y_{k}$ and $\Delta \theta_{k} \in[-\pi, \pi)$. Finally, $\alpha^{q_{k}}=\frac{a_{k}}{a_{k-1}}$, where we have $q_{k} \in\left[1-M_{a}, M_{a}-1\right]$.

The approximate form of the phase bit metric can be expressed with the aid of the exact a posteriori probability of
$\Upsilon_{y, k}$ and $\Delta \theta_{k}$ given $a_{k}, a_{k-1}, \psi_{k}$ and $\rho_{k}$, which is formulated as [10]:

$$
\begin{aligned}
& P_{P}\left(\Upsilon_{y, k}, \Delta \theta_{k} \mid a_{k}, a_{k-1}, \psi_{k}, \rho_{k}\right) \\
& \approx e^{-\frac{\left[\left|y_{k}\right|^{2}+\left|y_{k-1}\right|^{2} \Upsilon_{y, k}^{2}-2\left|y_{k}\right|\left|y_{k-1}\right| \Upsilon_{y, k} \cos \left(\Delta \theta_{k}-\psi_{k}\right)\right]}{N_{0}\left(1+\Upsilon_{y, k}^{2}\right)}} .
\end{aligned}
$$

2) Proposed M-DAPSK $\left(M_{a}, M_{p}\right)$ Soft Demapper: The soft-decision based M-DAPSK $\left(M_{a}, M_{p}\right)$ block is placed in front of the TC decoder, as portrayed in Fig. 1. The $k$ th received symbol may then be formulated as:

$$
y_{k}=h_{k} x_{k}+n_{k}=h_{k} a_{k} v_{k}+n_{k},
$$

where $h_{k}$ represents the non-dispersive Rayleigh fading coefficients, while $n_{k}$ represents the AWGN having a variance of $N_{0} / 2$ per dimension. For the sake of ensuring that two consecutive symbols experience a similar complex-valued fading envelop, which is a prerequisite for avoiding an errorfloor in differential detection, we assume a slowly Rayleigh fading channel, where we have $h_{k} \approx h_{k-1}$, based on (1), (6) can be rewritten as:

$$
\begin{aligned}
y_{k} & \approx h_{k-1} a_{k} v_{k-1} w_{k}+n_{k}, \\
& =\frac{a_{k}}{a_{k-1}}\left(y_{k-1}-n_{k-1}\right) w_{k}+n_{k}, \\
& =\frac{a_{k}}{a_{k-1}} y_{k-1} w_{k}+\tilde{n}_{k},
\end{aligned}
$$

where $\frac{a_{k}}{a_{k-1}}$ is the ratio of the $k$ th and $(k-1)$ st amplitudes, while

$$
\tilde{n}_{k}=-\frac{a_{k}}{a_{k-1}} n_{k-1} w_{k}+n_{k}
$$

is the effective noise ${ }^{1}$.

a) Amplitude Detection: $\left(2 M_{a}-1\right)$ amplitude ratios can be derived from the $M_{a}$-PSK ring radii of the MDAPSK $\left(M_{a}, M_{p}\right)$ scheme, which may be expressed as:

$$
\frac{a_{k}}{a_{k-1}}=\alpha^{b_{k}-b_{k-1}}=\alpha^{q_{k}},
$$

where $q_{k}$ obeys $\left(1-M_{a}\right) \leq q_{k} \leq\left(M_{a}-1\right)$.

${ }^{1}$ Since $-\frac{a_{k}}{a_{k-1}} w_{k}$ is a constant during a symbol period, the multiplication of the Gaussian noise $n_{k-1}$ by $-\frac{a_{k}}{a_{k-1}} w_{k}$ in (8) only affects the effective noise variance and the term $-\frac{a_{k}-1}{a_{k-1}} w_{k} n_{k-1}$ remains a Gaussian noise process. The sum of two Gaussian noise processes in (8) is also another Gaussian noise process, albeit associated with a different noise variance. 

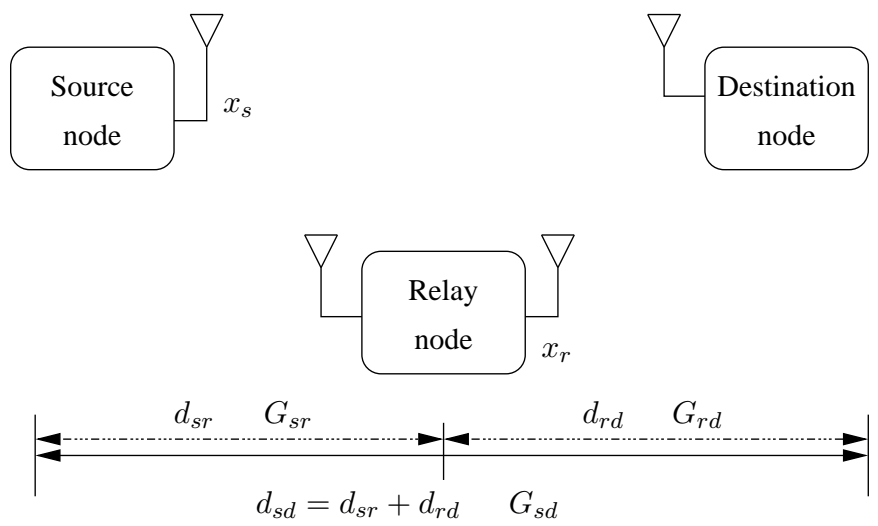

Fig. 2. The schematic of a two-hop relay-aided wireless system with S-D link

b) Probability Computation: The effective noise variance of $\tilde{n}_{k}$ in Eq. (7) depends on the amplitude ratio used at time instant $k$, which may be formulated as:

$$
\widetilde{N}_{0}=N_{0}+\alpha^{2 q_{k}} w_{k}^{2} N_{0}=N_{0}\left(1+\alpha^{2 q_{k}}\right),
$$

where we have $\widetilde{N}_{0}=\left(1+\alpha^{2 q_{k}}\right) N_{0}=N_{0}^{\left(q_{k}\right)}$. Based on (7) we have computed the probability of receiving $y_{k}$ conditioned on the transmission of $d_{k}$ and $f_{k}$ in (11) and (12), which are for the M-DAPSK $\left(2, M_{p}\right)$ scheme and for the M-DAPSK (4, $\left.M_{p}\right)$ scheme, respectively. The bit-probabilities may then be converted to the Log-Likelihood Ratio (LLR) [14] based representations of $c_{\mathrm{m} k+i}, 0 \leq i \leq(\mathrm{m}-1)$ :

$$
\begin{aligned}
P\left(y_{k} \mid d_{k}, f_{k}=0\right) & \approx e^{\frac{-\left|y_{k}-y_{k-1} \alpha^{0} \mu\left(d_{k}\right)\right|^{2}}{N_{0}^{(0)}}}, \\
P\left(y_{k} \mid d_{k}, f_{k}=1\right) & \approx e^{\frac{-\left|y_{k}-y_{k-1} \alpha^{-1} \mu\left(d_{k}\right)\right|^{2}}{N_{0}^{(-1)}}} \\
& +e^{\frac{-\left|y_{k}-y_{k-1} \alpha^{1} \mu\left(d_{k}\right)\right|^{2}}{N_{0}^{(1)}}} \cdot \\
P\left(y_{k} \mid d_{k}, f_{k}=0\right) & \approx e^{\frac{-\left|y_{k}-y_{k-1} \alpha^{0} \mu\left(d_{k}\right)\right|^{2}}{N_{0}^{(0)}}}, \\
P\left(y_{k} \mid d_{k}, f_{k}=1\right) & \approx e^{\frac{-\left|y_{k}-y_{k-1} \alpha^{-3} \mu\left(d_{k}\right)\right|^{2}}{N_{0}^{(-3)}}} \\
P\left(y_{k} \mid d_{k}, f_{k}=2\right) & \approx e^{\frac{-\left|y_{k}-y_{k-1} \alpha^{1} \mu\left(d_{k}\right)\right|^{2}}{N_{0}^{(1)}}} \\
& +e^{\frac{-\left|y_{k}-y_{k-1} \alpha^{-2} \mu\left(d_{k}\right)\right|^{2}}{N_{0}^{(-2)}}} \\
P\left(y_{k} \mid d_{k}, f_{k}=3\right) & \approx e^{\frac{\left.-\left|y_{k}-y_{k-1} \alpha^{2} \mu\left(y_{k}\right)\right|^{2} \alpha^{2}\right)\left.^{2} \mu\left(d_{k}\right)\right|^{2}}{N_{0}^{(2)}}} \\
& +e^{\frac{-\left|y_{k}-y_{k-1} \alpha^{3} \mu\left(d_{k}\right)\right|^{2}}{N_{0}^{(3)}}}
\end{aligned}
$$

\section{Amplify-and-Forward}

Fig. 2 presents the schematic of a two-hop relay-aided wireless system. According to the AF relaying protocol, the Relay Node (RN) amplifies the signal received from the Source
Node (SN) and forwards it to the Destination Node (DN) with the objective of achieving a more reliable transmission at a lower SNR when compared to the scheme dispensing with a RN. During the first time slot, the SN broadcasts $x_{s}$ to both the DN and RN. The kth symbol received at the DN may be expressed as:

$$
y_{s d, k}=\sqrt{G_{s d}} h_{s d, k} x_{s, k}+n_{s d, k},
$$

while the $k$ th symbol received at the RN could be formulated as:

$$
y_{s r, k}=\sqrt{G_{s r}} h_{s r, k} x_{s, k}+n_{s r, k},
$$

where $n_{s d, k}$ and $n_{s r, k}$ represents the AWGN having a variance of $N_{0} / 2$ per dimension, respectively. Furthermore, $h_{s d, k}$ and $h_{s r, k}$ represents the uncorrelated Rayleigh fading coefficient of the SD and SR links, respectively. Here, $G_{s r}=\left(\frac{d_{s d}}{d_{s r}}\right)^{2}$ is the reduced-distance-related pathloss reduction (RDRPLR) of the SR link with respect to the SD link [22], where $d_{a b}$ stands for the distance between node a and node $\mathrm{b}^{2}$. Similarly, we have $G_{s d}=1$.

The RN amplifies the signal received from the $\mathrm{SN}$ and forwards it to the DN by simply scaling the received signal by a factor that is inversely proportional to the received power, which can be formulated as:

$$
\tilde{\beta}_{f}=\frac{1}{\sqrt{G_{s r}\left|h_{s r}\right|^{2}+N_{0}}} .
$$

Since the average SNR at the RN's receiver may be expressed as:

$$
\gamma_{s r, k}=\mathrm{E}\left(\frac{G_{s r}\left|h_{s r, k}\right|^{2}}{N_{0}}\right),
$$

where $\mathrm{E}($.$) is the expectation of (.), the average value of \tilde{\beta}_{f}$ in Eq. (15) may be written as:

$$
\beta_{f}=\frac{1}{\sqrt{N_{0}} \sqrt{\gamma_{s r, k}+1}}
$$

Hence, the RN does not have to estimate the exact channel coefficient $h_{s r, k}$, when computing the amplification factor at the RN. Specifically, only the corresponding average received SNR $\gamma_{s r, k}$ is required, which is relatively easier to estimate. During the second transmission period, the RN amplifies the received signal as $\beta_{f} y_{s r}$ and forwards it to the DN. The $k t h$ symbol received at the DN may, therefore, be formulated as:

$$
\begin{aligned}
y_{r d, k} & =\sqrt{G_{r d}} h_{r d, k} x_{r, k}+n_{r d, k}, \\
& =\sqrt{G_{r d}} h_{r d, k} \beta_{f} y_{s r, k}+n_{r d, k}, \\
& =\underbrace{\sqrt{G_{r d}} h_{r d, k} \beta_{f} \sqrt{G_{s r}} h_{s r, k}}_{h_{s r d, k}} x_{s, k} \\
& +\underbrace{\sqrt{G_{r d}} h_{r d, k} \beta_{f} n_{s r, k}+n_{r d, k}}_{n_{s r d, k}},
\end{aligned}
$$

where $n_{r d, k}$ represents the AWGN having a variance of $N_{0} / 2$ per dimension, while $n_{s r d, k}$ is another Gaussian noise process with a different noise variance and $G_{r d}=\left(\frac{d_{s d}}{d_{r d}}\right)^{2}$.

${ }^{2}$ For the sake of simplicity we assumed without loss of generality that the $\mathrm{SN}$, the RN and the DN are positioned along a straight line in our paper, so we have $d_{s d}=d_{s r}+d_{r d}$ and $d_{s r}=d_{r d}=d_{s d} / 2$. 


\begin{tabular}{|l|r|}
\hline Modulation & 64-DAPSK (4,16), 32-DAPSK (4,8), 16-DAPSK (4,4) \\
8-DAPSK (4,2), 64-QAM, 64-DPSK
\end{tabular}

TABLE I

SYSTEM PARAMETERS.

According to Eq. (2), Eq. (18) may be rewritten as:

$$
y_{r d, k}=h_{s r d, k} a_{s, k} v_{s, k}+n_{s r d, k},
$$

and assuming a slow Rayleigh fading channel, where we have $h_{k} \approx h_{k-1}$, we arrive at:

$$
\begin{aligned}
y_{r d, k} & =h_{s r d, k-1} a_{s, k} v_{s, k-1} w_{k}+n_{s r d, k} \\
& =\alpha^{q_{k}} y_{r d, k-1} w_{k}-\underbrace{\alpha^{q_{k}} n_{s r d, k-1} w_{k}+n_{s r d, k}}_{\tilde{n}_{s r d, k}},
\end{aligned}
$$

while based on Eq. (18), $\tilde{n}_{s r d, k}$ may be expressed as:

$$
\begin{aligned}
\tilde{n}_{s r d, k} & =\sqrt{G_{r d}} h_{r d, k} \beta_{f} n_{s r, k}+n_{r d, k} \\
& -\alpha^{q_{k}} w_{k}\left(\sqrt{G_{r d}} h_{r d, k} \beta_{f} n_{s r, k}+n_{r d, k}\right) .
\end{aligned}
$$

The effective variance of the noise $\tilde{n}_{s r d, k}$ depends on the amplitude ratio $\alpha^{q_{k}}, G_{r d}, h_{r d}, k, \beta_{f}$ and $w_{k}$ which are constant during a symbol period. The effective noise variance is given by:

$$
N_{0}^{(i)}=N_{0}+N_{0}+\left|\alpha^{q_{k}}\right|^{2} N_{0}+\left|\alpha^{q_{k}}\right|^{2} N_{0},
$$

where the variance for each of the four noise terms in Eq. (22) is given in order by the right hand side of Eq. (23), respectively. Then, Eq. (23) may be substituted into Eq. (12) for the soft-decision M-DAPSK based on the AF relaying protocol.

\section{Simulation Results}

In this section, we characterize the performance of the proposed TC-aided soft-decision based M-DAPSK $\left(4, M_{p}\right)$ schemes. The classic square-constellation based 64-QAM and 64-DPSK schemes are used as benchmarks. The simulation parameters are shown in Table I.

Fig. 3 shows the BER versus SNR performance of both the BMIAD and of our proposed soft-decision 16-DAPSK $(2,8)$ schemes for transmission over correlated Rayleigh fading channels at different Doppler frequencies $f_{d}$ and different number of symbols $L_{s f}$ per 64-DAPSK block. Observe in

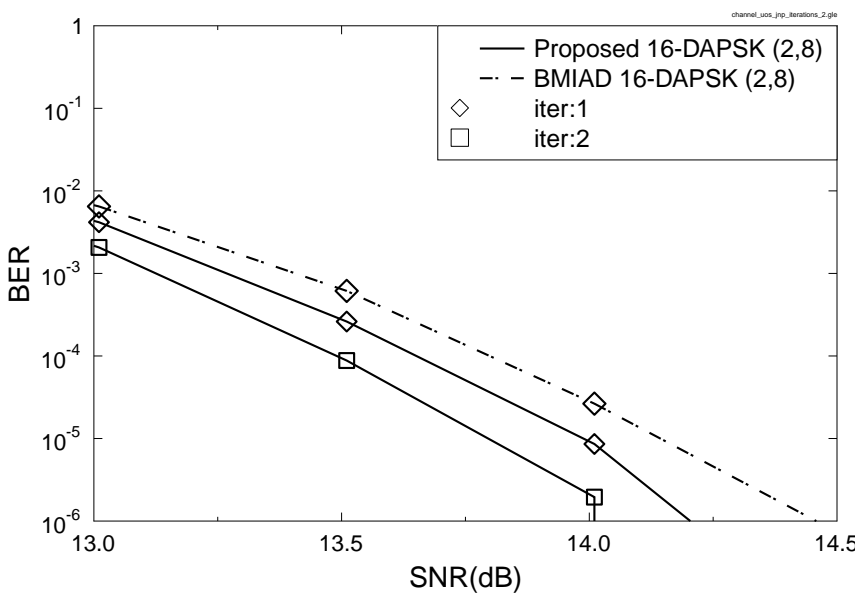

Fig. 4. BER versus SNR (dB) performance comparison of the BMIAD 16-DAPSK $(2,8)$ and proposed soft-decision 16-DAPSK $(2,8)$ schemes for transmission over correlated Rayleigh fading channels with $f_{d}=0.01$ and different outer iteration numbers. The corresponding system parameters are summarized in Table I

Fig. 3 that our proposed soft-decision aided 16-DAPSK $(2,8)$ scheme achieves a $0.3 \mathrm{~dB}$ SNR gain at BER $=10^{-5}$, compared to the BMIAD 16-DAPSK $(2,8)$ scheme. When $L_{s f}=10$, the BER performance seen in Fig. 3(a) is similar to that in Fig. 3(b), but upon increasing $L_{s f}$, the BER differences become more obvious. Furthermore, as seen in Fig. 3(b), the BER performance of the $L_{s f}=100$ scenario and of the $L_{s f}=400$ scenario is similar for our proposed softdecision aided 16-DAPSK $(2,8)$ scheme, when transmitting over correlated Rayleigh fading channels associated with $f_{d}=0.01$. Hence, $f_{d}=0.01$ and $L_{s f}=400$ are employed in the following simulations. The other advantage of our proposed soft-decision 16-DAPSK $(2,8)$ scheme is evidenced by Fig. 4, the non-iterative BMIAD aided 16-DAPSK-TC arrangement (the diamonded-dotted curve) is considered as our benchmark scheme. The iterations between the demapper and the decoder are also capable of improving the BER performance. More specifically, after the second iteration the 16-DAPSK $(2,8)$-TC outperforms the noniterative 16-DAPSKTC by approximately $0.2 \mathrm{~dB}$.

The EXIT Charts of the 64-QAM, 64-DAPSK $(4,16)$ and 64-DPSK aided TC schemes recorded, when communicating over a correlated Rayleigh channel at $\mathrm{SNR}=16 \mathrm{~dB}$ are shown in Fig. 5. The SNR-independent EXIT curve of the outer TC decoder is also shown. More specifically, the dashed and unmarked curves are the EXIT curves of the inner decoder, namely those of the 64-QAM, 64-DAPSK $(4,16)$ and 64DPSK schemes with Gray labeling, respectively. Moreover, the dark-circled solid curve stands for the EXIT curve of the outer TC decoder, while the circled-dashed line is that of the outer convolutional decoder (CC). According to [15], [23], [24], the area under the EXIT curve of the inner decoder is approximately equal to the channel capacity. It is clear that the area under the square-constellation 64-QAM scheme's EXIT curve is the largest, while that of the 64-DPSK is the smallest. It can be seen in Fig. 5 that the area under the squareconstellation 64-QAM scheme's EXIT curve is larger than that under the 64-DAPSK $(4,16)$ scheme's curve, which is in turn higher than that of the 64-DPSK arrangement. In both cases 


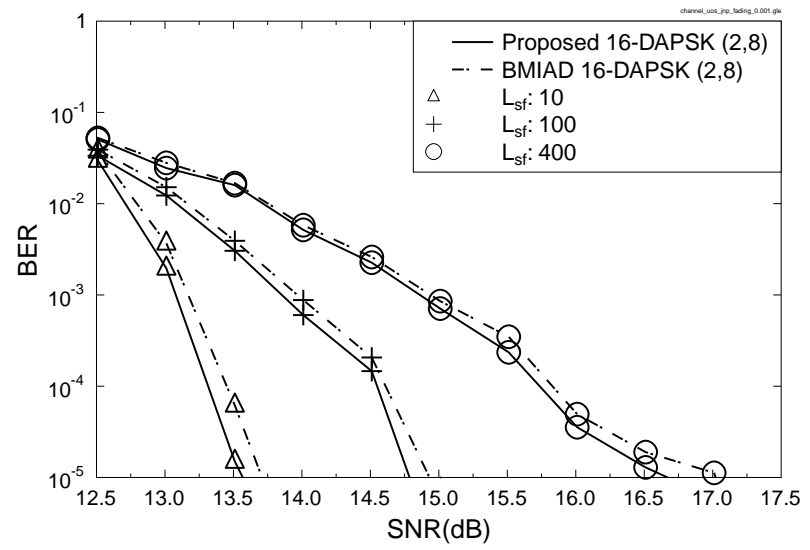

(a) $f_{d}=0.001$

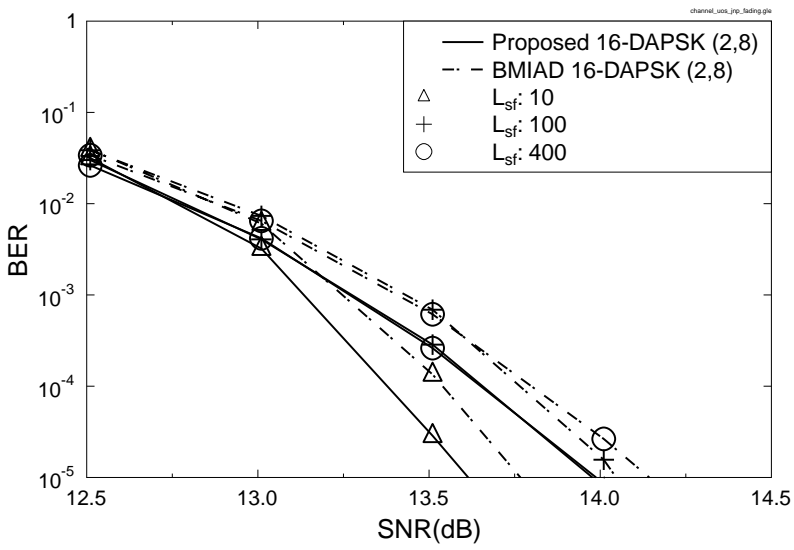

(b) $f_{d}=0.01$

Fig. 3. The comparison of the BMIAD and our proposed soft-decision demapper for the 16-DAPSK $(2,8)$ system for transmission over correlated Rayleigh fading channels with different $f_{d}$ and different $L_{s f}$, while the outer iterations being 1 . The rest of the corresponding system parameters are summarized in Table I.

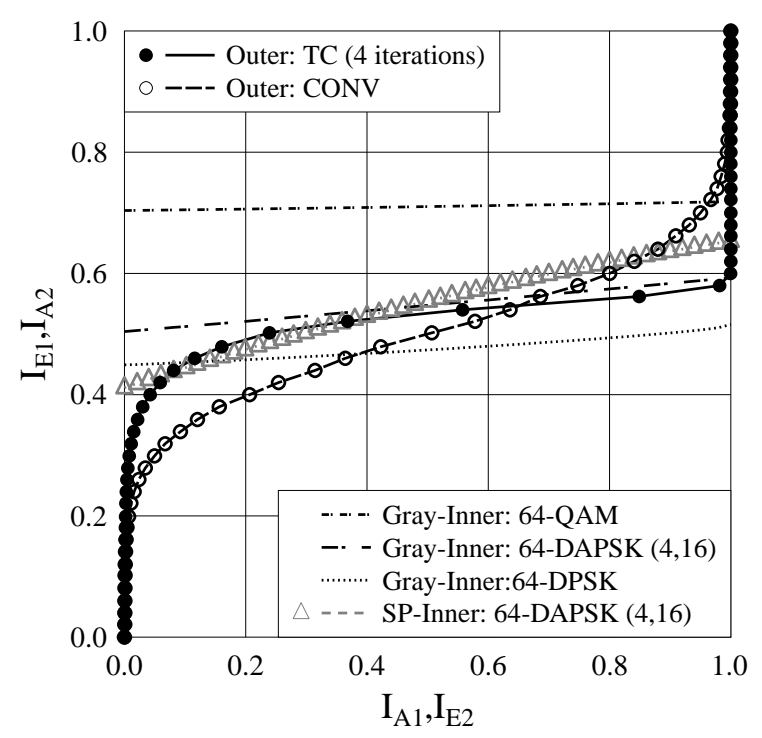

Fig. 5. EXIT Charts of the 64-QAM, 64-DAPSK $(4,16)$ and 64-DPSK aided TC schemes when communicating over a correlated Rayleigh channel at $\mathrm{SNR}=16 \mathrm{~dB}$. The SNR-independent EXIT curve of the outer TC decoder is also shown. The corresponding simulation parameters are presented in Table I.

an open is observed tunnel between the inner curves and the outer curves indicating, that convergence is possible at this SNR. Note that only the EXIT function of the inner decoder depends on the SNR and an open EXIT chart tunnel implies having an infinitesimally low BER [14]. Hence we may argue based on Fig. 5 that a vanishingly low BER may be achieved by the TC aided 64-DAPSK $(4,16)$ scheme for SNR values in excess of $16 \mathrm{~dB}$. By contrast, no open EXIT chart tunnel is maintained for the same SNR value in the case of the 64DPSK benchmark scheme. Note that the EXIT curve of the CC does not match that of the 64-DAPSK $(4,16)$ demapper, while that of the TC does. Furthermore ${ }^{3}$, the triangle-dotted

\footnotetext{
${ }^{3}$ We employ a 64-state CC here, which has the same complexity as that of the TC scheme. The total number of trellis states in a TC scheme that employs two 8 -state component codes and $4 \mathrm{TC}$ iterations is given by $2 \times 8 \times 4=64$. It is possible to match the EXIT curve of a CC scheme that employs a very high number of trellis states [24] to the demapper's EXIT curve, but it is not practical and unfair to do so.
}

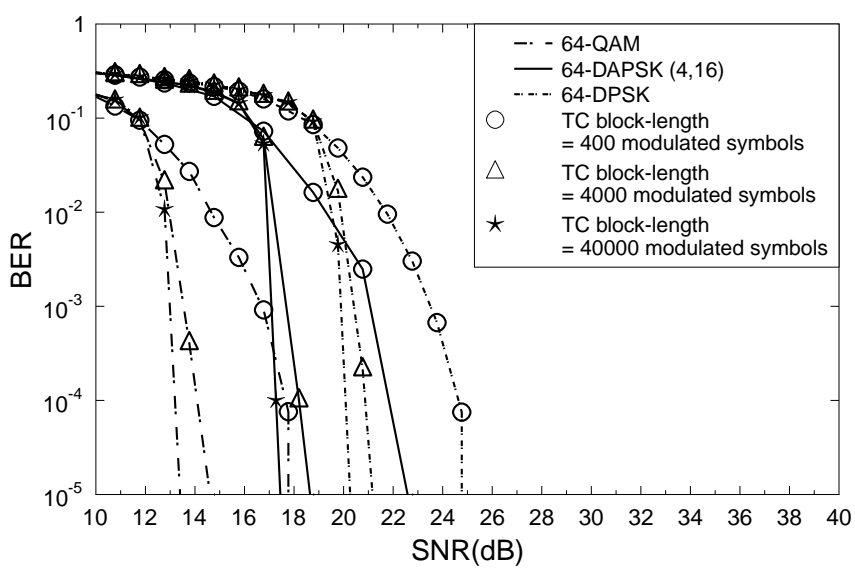

Fig. 6. BER versus SNR (dB) performance comparison of the 64-QAM, 64-DAPSK $(4,16)$ and 64-QAM schemes for transmission over correlated Rayleigh fading channels. The corresponding system parameters are summarized in Table I. A TC block-length of 400 modulated symbols corresponds to one 64-DAPSK block-length, while a 4000-modulated-symbol TC block corresponds to ten 64-DAPSK block-length.

curve shows the EXIT Charts of the 64-DAPSK $(4,16)$ aided TC schemes with Set Patitioning (SP) labeling method. It can be seen from Fig. 5 that the EXIT curve of the Gray labeling scheme matches that of the TC scheme at $\mathrm{SNR}=16 \mathrm{~dB}$, while the EXIT curve of the SP labelled scheme intersects with that of the TC scheme.

Fig. 6 shows the corresponding BER versus SNR performance, which compares the performance of the TC-aided 64QAM, 64-DAPSK $(4,16)$ and 64-DPSK aided TC schemes, when communicating over correlated Rayleigh fading channels using different transmission block lengths ${ }^{4}$ and turbointerleaved block lengths (Table I). When the number of 64DAPSK $(4,16)$ modulated transmission blocks per TC block is one, which corresponds to the curve marked by circles in Fig. 6, the SNR difference between the classic coherently detected square-constellation 64-QAM and our low-complexity

\footnotetext{
${ }^{4}$ In our paper, the TC block length is given by the number of modulated symbols per 64-DAPSK $(4,16)$ transmission block times the number of transmission blocks per TC block.
} 


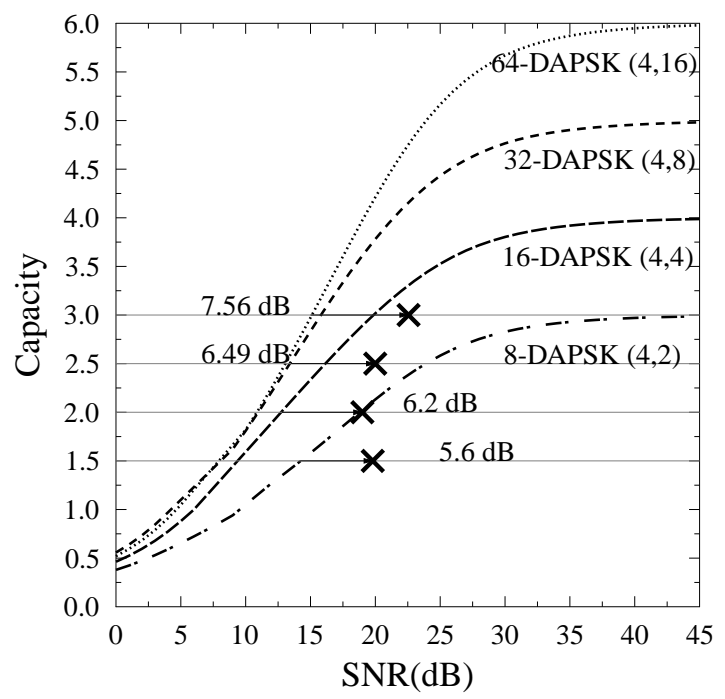

(a) TC block length of 400 modulated symbols.

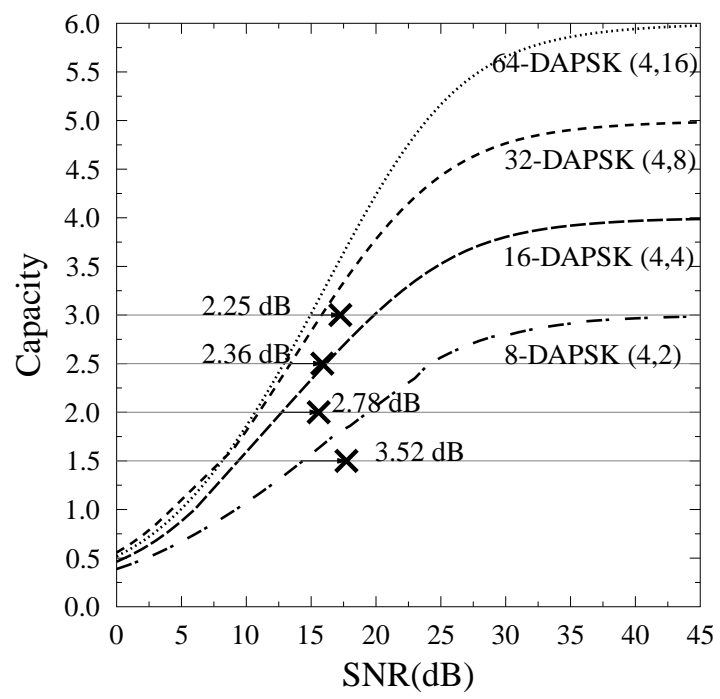

(b) TC block length of 40,000 modulated symbols.

Fig. 7. Achievable throughput versus SNR (dB) for transmission over correlated Rayleigh fading channels, while using different TC block length.

64-DAPSK $(4,16)$ dispensing with channel-estimation is 4.35 dB. As a substantial further benefit, our scheme outperforms 64-DPSK by about $2.3 \mathrm{~dB}$. Moreover, when the number of 64-DAPSK $(4,16)$ blocks per TC block is increased to one hundred (the curve marked by star), all the BER performances are improved. Compared to the scenario, when the number of 64-DAPSK $(4,16)$ blocks per TC block is one, the SNR gain of the arrangement having 100 blocks per TC block is improved by $5.25 \mathrm{~dB}$. In general, the longer the TC block-length, the closer the BER performance curve to the channel capacity.

Fig. 7 quantifies the maximum achievable throughput of various M-DAPSK $\left(4, M_{p}\right)$ schemes, where the curves were generated by evaluating the area under the corresponding EXIT curves, as mentioned above and detailed in [14]. The horizontal dotted lines represent the throughput values of the different turbo-coded modulation schemes considered. More explicitly, 1.5, 2.0, 2.5 and 3.0 bits/symbol are the throughputs that of TC aided 8-DAPSK $(4,2), 16-$ DAPSK $(4,4) 32$ DAPSK $(4,8) 64$-DAPSK $(4,16)$, respectively. Each large cross is located at the SNR required for the corresponding TCaided modulation scheme to achieve an identical throughput to each other at a target BER of $10^{-5}$. The SNR values shown next to the large crosses indicate the distances to the corresponding channel capacity. Fig. 7(a) presents the achievable throughput versus SNR (dB) at a TC block length of 400 modulated symbols for the various TC-aided 64 DAPSK $(4$, $m_{p}$ ) schemes are capable of operating within $7.56 \mathrm{~dB}$ from their corresponding capacity curves. When using a longer TC block length of 40000 modulated symbols, the various TCaided M DAPSK $\left(4, m_{p}\right)$ schemes are capable of operating within $2.25 \mathrm{~dB}$ from their corresponding capacity curves, as shown in Fig. 7(b). Hence, as expected, the larger the TC block size employed, the closer the system operates to capacity. The large crosses represent the SNR required for the corresponding

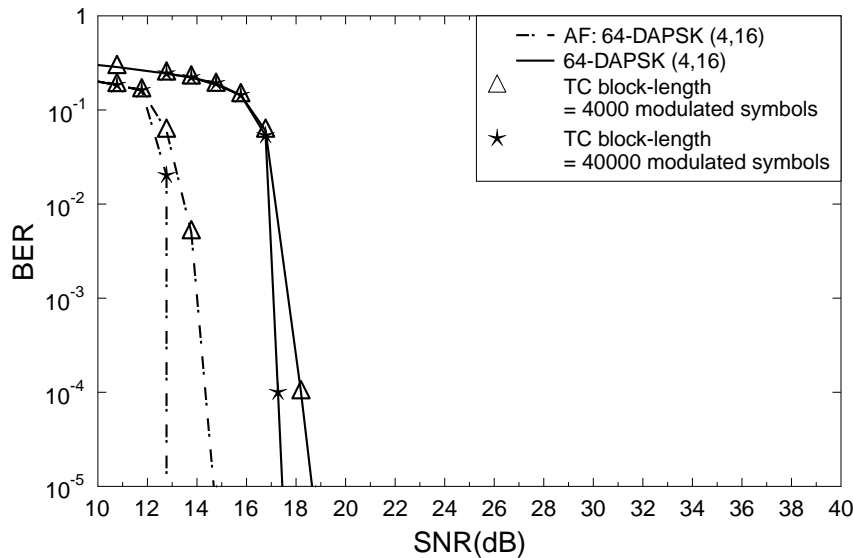

Fig. 8. BER versus SNR (dB) performance comparison of the 64DAPSK $(4,16)$ schemes when transmitting over correlated Rayleigh fading channels. Both non-cooperative scheme and AF-based cooperative scheme are considered. The corresponding system parameters are summarized in Table I. A TC block-length of 4000 modulated symbols corresponds to ten 64-DAPSK block-length, while a 40000-modulated-symbol TC block corresponds to one hundred 64-DAPSK block-length.

modulation schemes to achieve an identical throughput to each other at a target BER of $10^{-5}$.

Fig. 8 presents the BER versus SNR performance of the soft-decision 64-DAPSK $(4,16)$ scheme and that invoking the $\mathrm{AF}$ protocol, when the $\mathrm{RN}$ is located in the middle of the SN-DN link. As indicated in Fig. 8, the BER performance recorded for the AF protocol is significantly better than that of 64-DAPSK for the non-cooperative system. For example, when using a TC block length of 40000 modulated symbols, the SNR gain of the TC-aided 64-DAPSK $(4,16)$ under the $\mathrm{AF}$ relaying protocol is improved by $4.5 \mathrm{~dB}$ at $\mathrm{BER}=10^{-5}$, when compared to the non-cooperative scenario. 


\section{CONCLUSIONS}

In this treatise, we firstly compared the BMIAD and our proposed soft-decision demapper for the 16-DAPSK $(2,8)$ design example. Then we generalised the symbol-to-bit softdemapper probability formulas of the M-DAPSK $\left(M_{a}, M_{p}\right)$ scheme. EXIT charts were used for quantifying the achievable DCMC capacity of the various TC-aided M-DAPSK $\left(M_{a}, M_{p}\right)$ modulation schemes. The 64-DAPSK-TC scheme outperforms the identical-throughput 64-DPSK-TC scheme by about $2.3 \mathrm{~dB}$ at a BER of $10^{-5}$, when communicating over correlated Rayleigh fading channels having a normalised Doppler frequency of 0.01 and a TC block length of 40000 modulated symbols. The SNR distance of the 64-DAPSK-TC scheme from the capacity is $2.25 \mathrm{~dB}$. Finally, we have proposed the soft-decision M-DAPSK aided AF based relaying scheme, which is capable of attaining a further $4.5 \mathrm{~dB}$ SNR gain. Our future research will consider further reducing the complexity of our TC-aided M-DAPSK $\left(M_{a}, M_{p}\right)$ scheme.

\section{REFERENCES}

[1] D. Liang, S. X. Ng, and L. Hanzo, "Near-capacity turbo coded softdecision aided DAPSK/Star-QAM," in IEEE Veh. Technol. Conf., San Francisco, USA, 5-8 Sept. 2011.

[2] E. Issman and W. Webb, "Carrier recovery for 16-level QAM in mobile radio," IEE Colloquium Multi-Level Modulation, pp. 9/1 - 9/8, Mar. 1990.

[3] L. Chen, H. Kusaka, and M. Kominami, "Blind phase recovery in QAM communication systems using higher order statistics," IEEE Signal Process. Lett., vol. 3, no. 5, pp. 147-149, May. 1996.

[4] Y. Wang and E. Serpedin, "A class of blind phase recovery techniques for higher order QAM modulations: Estimators and bounds," IEEE Signal Process. Lett., vol. 9, no. 10, pp. 301-304, Oct. 2002.

[5] H. Rohling and V. Engels, "Differential amplitude phase shift keying (DAPSK)-a new modulation method for DTVB," in Broadcasting Convention, 1995. IBC 95., International, Sept. 1995, pp. 102-108.

[6] W. Webb, L. Hanzo, and R. Steele, "Bandwidth-efficient QAM schemes for Rayleigh-fading channels," IEE Proc., vol. 138, no. 3, pp. 169-175, June 1991.

[7] S.-I. Chen and T. Fuja, "Soft-decision decoding metrics for DAPSK," in Proc. IEEE International Symp. Inf. Theory, June 1997, p. 304.

[8] T. May, H. Rohling, and V. Engels, "Performance analysis of viterbi decoding for 64-DAPSK and 64-QAM modulated OFDM signals," IEEE Trans. Commun., vol. 46, no. 2, pp. 182-190, Feb. 1998.

[9] B. Eitel and J. Speidel, "Speed-optimized soft-decision demodulation of multilevel DAPSK," in International Conf. Consumer Electron. Technical Papers, Jan. 2006, pp. 469-470.

[10] K. Ishibashi, H. Ochiai, and R. Kohno, "Low-complexity bit-interleaved coded dapsk for Rayleigh-fading channels," IEEE J. Sel. Areas Commun., vol. 23, no. 9, pp. 1728-1738, Sept. 2005.

[11] D. Liang, S. X. Ng, and L. Hanzo, "Soft-decision Star-QAM aided BICM-ID," Signal Process. Lett., IEEE, vol. 18, no. 3, pp. 169-172, 2011.

[12] C. Berrou and A. Glavieux and P. Thitimajshima, "Near Shannon limit error-correcting coding and decoding: Turbo codes," in Proc. International Conf. Commun., Geneva, Switzerland, May 1993, pp. 1064-1070.

[13] C. Berrou and A. Glavieux, "Near optimum error correcting coding and decoding: Turbo-codes," IEEE Trans. Commun., vol. 44, no. 10, pp. 1261-1271, Oct. 1996.

[14] L. Hanzo, T. H. Liew, B. L. Yeap, R. Y. S. Tee, S. X. Ng, Turbo Coding, Turbo Equalisation and Space-Time Coding: EXIT-Chart-Aided Near-Capacity Designs for Wireless Channels, 2nd Edition. Wiley-IEEE Press, 2011.

[15] S. Ten Brink, "Convergence behavior of iteratively decoded parallel concatenated codes," IEEE Trans. Commun., vol. 49, no. 10, pp. $1727-$ 1737, Oct. 2001

[16] A. Sendonaris, E. Erkip, and B. Aazhang, "User cooperation diversity. Part i. System description," IEEE Trans. Commun., vol. 51, no. 11, pp. 1927-1938, Nov. 2003.

[17] J. Laneman, D. Tse, and G. Wornell, "Cooperative diversity in wireless networks: Efficient protocols and outage behavior," IEEE Trans. Inf. Theory, vol. 50, no. 12, pp. 3062-3080, Dec. 2004.
[18] Q. Zhao and H. Li, "Differential modulation for cooperative wireless systems," IEEE Trans. Signal Process., vol. 55, no. 5, pp. 2273-2283, May 2007.

[19] T. Himsoon, W. Siriwongpairat, W. Su, and K. Liu, "Differential modulations for multinode cooperative communications," IEEE Trans. Signal Process., vol. 56, no. 7, pp. 2941-2956, July 2008.

[20] C.-H. Huang and C.-D. Chung, "Differentially amplitude- and phaseencoded qam for amplify-and-forward multiple-relay systems," IEEE Trans. Veh. Technol., vol. 61, no. 5, pp. 2054-2066, June 2012.

[21] M. Rohling, T. May, K. Bruninghaus, and R. Grunheid, "Broad-band OFDM radio transmission for multimedia applications," Proc. IEEE, vol. 87 , no. 10, pp. 1778-1789, Oct. 1999.

[22] H. Ochiai, P. Mitran, and V. Tarokh, "Design and analysis of collaborative diversity protocols for wireless sensor networks," in IEEE Veh. Technol. Conf., Sept. 2004, pp. 4645-4649.

[23] J. Kliewer, S. X. Ng, and L. Hanzo, "Efficient computation of EXIT functions for non-binary iterative decoding," IEEE Trans. Commun., vol. 54, no. 12, pp. 2133-2136, Dec. 2006.

[24] S. X. Ng, J. Wang and L. Hanzo, "Unveiling near-capacity code design: The realization of Shannon's communication theory for MIMO channels," in IEEE ICC 2008, Beijing, China, 19-23 May 2008, pp. $1415-1419$.

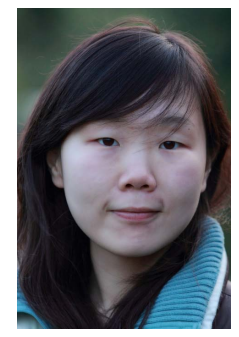

Dandan Liang received her B.Eng. degree (First class) in electronic science and technology from the PLA Information Engineering University, Zhengzhou, China, in 2008 and M.Sc. degree (First class) in radio frequency communication systems from the University of Southampton, UK, in 2009. She is currently working towards the $\mathrm{PhD}$ degree with the Research Group of Communications, Signal Processing and Control, School of Electronics and Computer Science, University of Southampton, UK. Her research interests include adaptive coded modulation, coded modulation, non/coherent modulation detection, iterative detection, networking coding, cooperative communications as well as wirelessoptical fiber communications.

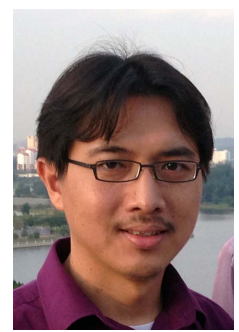

Dr Soon Xin Ng (S'99-M'03-SM'08) received the B.Eng. degree (First class) in electronics engineering and the Ph.D. degree in wireless communications from the University of Southampton, Southampton, U.K., in 1999 and 2002, respectively. From 2003 to 2006, he was a postdoctoral research fellow working on collaborative European research projects known as SCOUT, NEWCOM and PHOENIX. Since August 2006, he has been a member of academic staff in the School of Electronics and Computer Science, University of Southampton. He is involved in the OPTIMIX and CONCERTO European projects as well as the IU-ATC and UC4G projects. He is currently a senior lecturer at the University of Southampton.

His research interests include adaptive coded modulation, coded modulation, channel coding, space-time coding, joint source and channel coding, iterative detection, OFDM, MIMO, cooperative communications, distributed coding, quantum error correction codes and joint wireless-and-optical-fiber communications. He has published over 150 papers and co-authored two John Wiley/IEEE Press books in this field. He is a senior member of the IEEE, a Chartered Engineer and a fellow of the Higher Education Academy in the UK. 


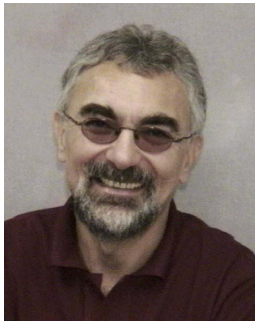

Lajos Hanzo FREng, FIEEE, FIET, Fellow of EURASIP, DSc received his degree in electronics in 1976 and his doctorate in 1983. In 2009 he was awarded the honorary doctorate "Doctor Honoris Causa" by the Technical University of Budapest. During his 35-year career in telecommunications he has held various research and academic posts in Hungary, Germany and the UK. Since 1986 he has been with the School of Electronics and Computer Science, University of Southampton, UK, where he holds the chair in telecommunications. He has successfully supervised $80 \mathrm{PhD}$ students, co-authored 20 John Wiley/IEEE Press books on mobile radio communications totalling in excess of 10000 pages, published 1300 research entries at IEEE Xplore, acted both as TPC and General Chair of IEEE conferences, presented keynote lectures and has been awarded a number of distinctions. Currently he is directing a 100-strong academic research team, working on a range of research projects in the field of wireless multimedia communications sponsored by industry, the Engineering and Physical Sciences Research Council (EPSRC) UK, the European IST Programme and the Mobile Virtual Centre of Excellence (VCE), UK. He is an enthusiastic supporter of industrial and academic liaison and he offers a range of industrial courses. He is also a Governor of the IEEE VTS. During 2008 - 2012 he was the Editor-in-Chief of the IEEE Press and a Chaired Professor also at Tsinghua University, Beijing. His research is funded by the European Research Council's Senior Research Fellow Grant. For further information on research in progress and associated publications please refer to http://www-mobile.ecs.soton.ac.uk. 